\title{
Rheology of biofilms formed from the dental plaque pathogen Streptococcus mutans
}

\author{
* Corresponding author: \\ Dr P. Stoodley \\ Departments of Microbiology and Civil \\ Engineering, Montana State \\ University-Bozeman, Bozeman \\ MT 59717, USA \\ T 14069947361 \\ F 14069946098 \\ E paul_s@erc.montana.edu \\ 1 Department of Mechanical and \\ Industrial Engineering, Montana State \\ University-Bozeman, Bozeman, \\ MT 59717, USA \\ 2 Center for Biofilm Engineering, \\ Montana State University-Bozeman, \\ Bozeman, MT 59717, USA \\ 3 Departments of Microbiology and \\ Civil Engineering, Montana State \\ University-Bozeman, Bozeman, \\ MT 59717, USA
}

\author{
A. M. Vinogradov', M. Winston ${ }^{1,2}$, C. J. Rupp ${ }^{1,2}$ \\ and P. Stoodley ${ }^{3 *}$
}

\begin{abstract}
Here we describe an experimental study of the mechanical properties of bacterial biofilms formed from the early dental plaque colonizer Streptococcus mutans. The $S$. mutans biofilms demonstrated the behavior of rheological fluids, with properties similar to those of organic polymers and other biological fluids. The time-dependent response of the biofilms was modeled on the basis of principles of viscoelasticity theory. The static and dynamic responses were defined in terms of the creep compliance, storage and loss moduli, and viscosity. The creep compliance and stress relaxation functions of $S$. mutans biofilms were characterized using the Burger model. Implications for developing more effective mechanical removal strategies of dental plaque biofilms are discussed.
\end{abstract}

\section{INTRODUCTION}

The mechanical removal of biofilms is a cornerstone in oral healthcare. Dental plaque is most commonly removed through routine tooth brushing and flossing (Gift, 1986), in which removal is achieved primarily through applied shear forces generated through direct contact of toothbrush bristles and the scouring action of the bristles across tooth and gum surfaces. Twice daily brushing and flossing continually disrupts the natural succession of biofilms from primary colonizers to a mature plaque, which may harbor pathogens responsible for cavities (VanHoute, 1980; Kuramitsu, 1993), gingivitis and, eventually, periodontal disease (Moore et al., 1987).

In traditional brushing, the forces necessary to remove the plaque are generated by manual scrubbing, which provides little control over the magnitude and manner in which these forces are applied. As a result, the effectiveness of the plaque removal is highly user dependent. The introduction of powered brushes tends to improve the control over the generated forces. Since mechanical brushing is characterized by various parameters such as speed, lateral frequency and amplitude, the entire process can be precisely programmed, leading to increased effectiveness of biofilm removal. In addition to traction generated through physical contact, in vitro studies have demonstrated that significant removal of a Streptococcus mutans biofilm could be achieved through the generation of local turbulence and shear, even when the brush was $1 \mathrm{~mm}$ away from the biofilm (Adams et al., 2002; Heersink et al., 2003). Significant in vitro effects from shear forces and surface tension on the detachment of dental plaque biofilms have also been reported (Christersson et al., 1988, 1989).

The ability to control biofilm removal through the use of powered brushes opens up the possibility of optimizing the practicality and effectiveness of this method. In this regard, it is important to understand the mechanical response of biofilms to applied external forces under various loading conditions, which, considering the present lack of knowledge, is a challenging task. Several recent publications demonstrate that biofilms can be treated as viscoelastic fluids (Stoodley et al., 1999a,b; Koerstgens et al., 2001; Klapper et al., 2002; Towler et al., 2003).

The objective of this study was to investigate the mechanical properties of biofilms formed from S. mutans, an early colonizer of hard tooth surfaces implicated in acid production and cavity formation (Marsh, 2000). The biofilms were tested using a TA Instruments AR 1000 shear-controlled rheometer with protocols that we had developed for testing pond water biofilms (Towler et al., 2003). We tested the biofilms under shear, since shear is often the principal force acting on biofilms growing in flowing fluids, and shear forces generated by sonicpowered brushing are being utilized for biofilm removal in areas beyond bristle contact (Heersink et al., 2003). Biofilms were grown and tested non-destructively directly on hydroxyapatite-coated parallel disk rheometer plates. Parallel plates geometries have several advantages for biofilm measurements over the more standard "cone and plate" or "concentric ring" geometries. First, the 


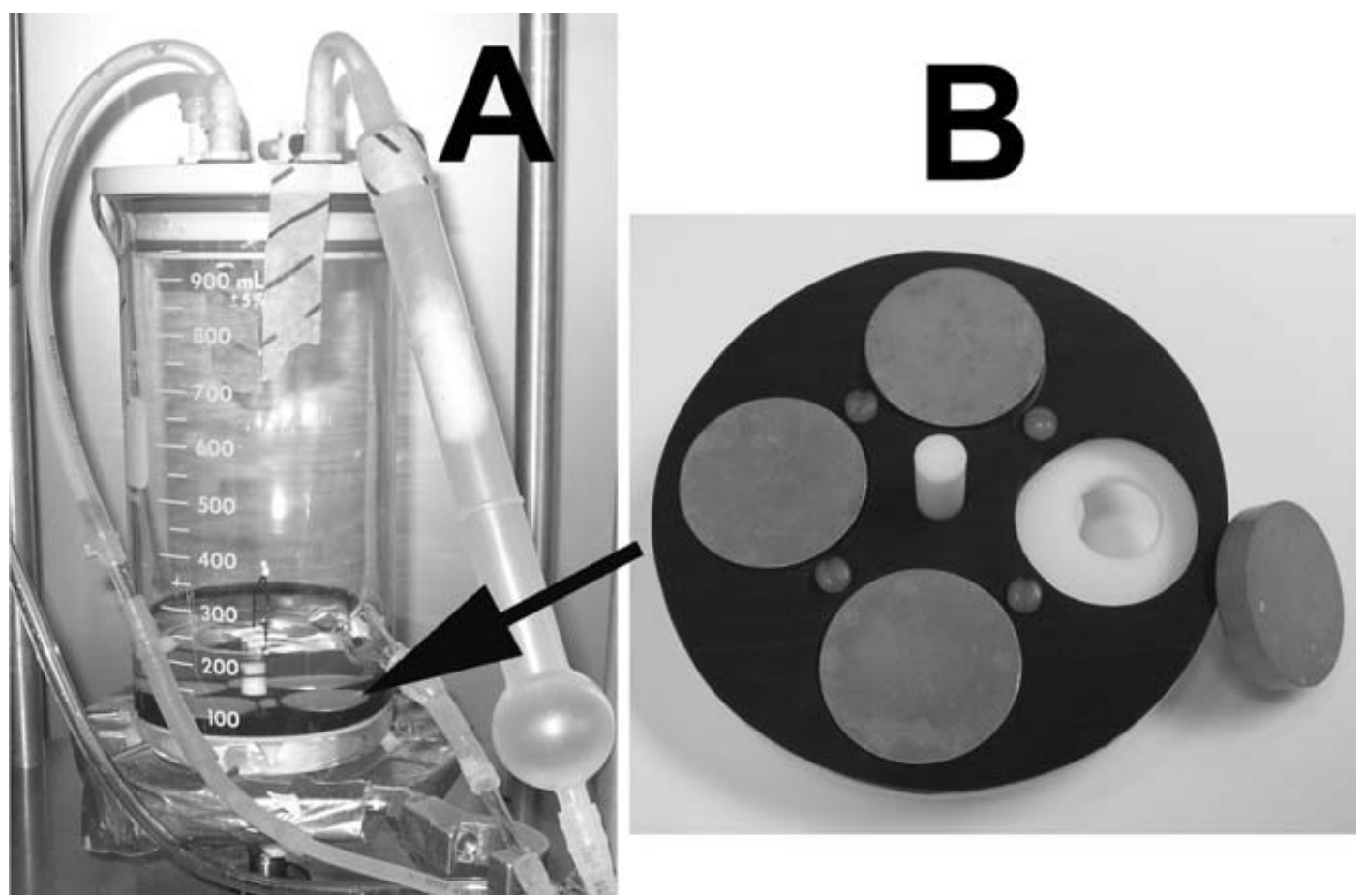

Fig. 1: (A) Rotating disk reactor (RDR) showing nutrient and $\mathrm{CO}_{2}$ inlet tubes, overflow effluent port and rotor plate (indicated by arrow). (B) Close-up of rotor plate with three $\mathrm{HA}$-coated rheometer disks in operating position and one disk placed to the side.

gap thickness can be adjusted to accommodate biofilms of variable thickness. Secondly, the flat surface is more conducive for growing biofilms uniformly over the geometry. Thirdly, flat geometries are more compatible for positioning in standard biofilm culturing apparatus such as flow cells and chemostat type reactors. The experimental results were characterized in terms of the basic principles of viscoelasticity theory.

\section{MATERIALS AND METHODS}

\section{Growth of biofilm samples}

Biofilms were grown in a BioSurface Technologies rotating disk reactor (RDR) (Fig. 1A) according to ASTM (American Society for Testing and Materials) standard method E-2196-02. The RDR consisted of a rotor plate, $1000 \mathrm{ml}$ chemostat and a magnetic stir plate. The rotor plate was imbedded with a drive magnet, allowing continuous rotation of the RDR rotor (Fig. 1b) at $100 \pm 20$ r.p.m. (Fig. 1B). The rotor plate was modified to accept four $25 \mathrm{~mm}$ diameter anodized, parallel, flat plate rheometer disks. The disks were coated with hydroxyapatite (HA) (Clarkson Chromatography Products Inc., Williamsport, PA). The thickness and surface coverage of the HA was $3.1 \pm 1.4 \mu \mathrm{m}$ and $87 \pm 11 \%$ respectively ( $n=15$, five locations from each of three disks) measured with epifluorescent microscopy using the autofluorescence of the HA. The rheometer disks were centered at a radial distance of $2.25 \mathrm{~cm}$ from the center of the rotor plate. During operation the fluid depth above the surfaces of the rheometer disks was approximately $2 \mathrm{~cm}$. The chemostat was equipped with nutrient and gas inlets at the top, and an effluent outlet at the bottom. Nutrients were fed to the system using a peristaltic pump (Cole-Parmer model no. 7553-80). $\mathrm{A} \mathrm{CO}_{2}$ headspace was maintained through the gas inlet.

\section{Inoculum and medium}

To emulate early dental biofilms, S. mutans UA159 (American Type Culture Collection (ATCC) no. 700610: $<$ www.atcc.org $>$ ) were grown on the HA-coated rheometer disks, using DIFCO ${ }^{\complement}$ Brain Heart Infusion media (BHI). Frozen stock culture $(0.5 \mathrm{ml})$ was used to inoculate $125 \mathrm{ml}$ of full strength $(37 \mathrm{~g} / \mathrm{l}) \mathrm{BHI}$ and grown anaerobically, using $\mathrm{CO}_{2}$ capsules, in a $37^{\circ} \mathrm{C}$ incubator for $24 \mathrm{~h}$. A $4 \mathrm{ml}$ volume of this batch culture was used to inoculate $250 \mathrm{ml}$ of $\mathrm{BHI}$ in the RDR. For the initial $24 \mathrm{~h}$ the RDR was operated in batch culture mode to allow cells to attach. The RDR was then switched to continuous culture (nutrient flow rate $7 \mathrm{ml} / \mathrm{min}$ ) using one-tenth strength BHI for a period of $48 \mathrm{~h}$. During operation, the liquid volume of the RDR was $170 \mathrm{ml}$, giving a resulting residence time of $24 \mathrm{~min}$. After the growth period the rheometer disks were removed and placed in Ringers buffer solution for either rheological analysis or microscopic inspection. 

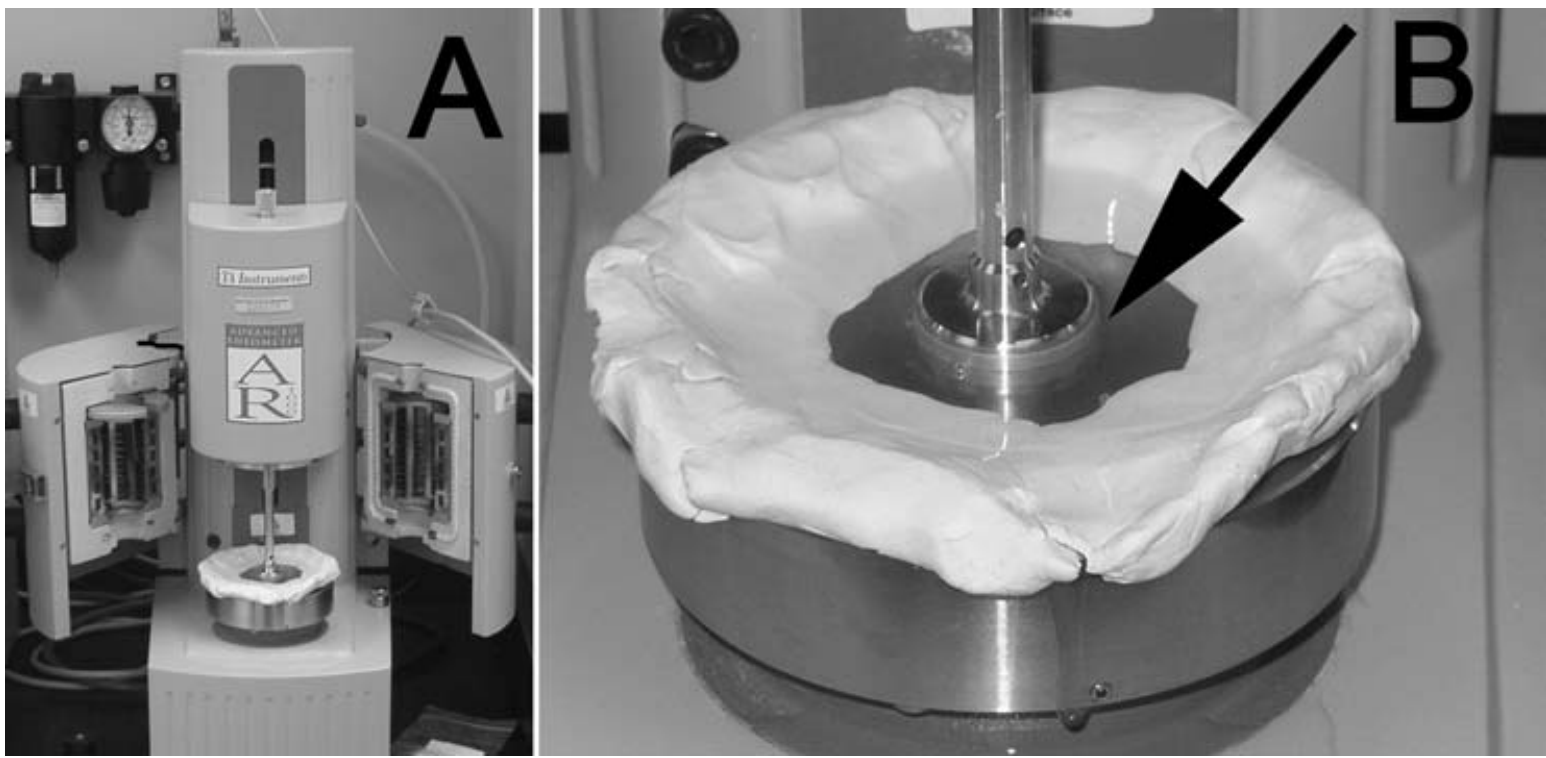

Fig. 2: (A) The AR 1000 rheometer with a HA-coated disk colonized with biofilm in testing mode. (B) Close up of the disk (indicated by arrow). A dam of modeling clay was used to allow the shear testing to be conducted in submersion.

\section{Rheometry}

Biofilms were tested using a TA Instruments AR 1000 Rheometer either (a) by quasi-static creep tests under the conditions of constant shear stresses or (b) by dynamic tests of biofilms subjected to sinusoidal oscillations. To prevent dehydration of the biofilm during testing and simulate the growth environment, the tests were performed in submersion by fashioning a water dam around the edge of the AR1000 base-plate, using modeling clay (Fig. 2). To position a biofilm sample in the rheometer, the disks were threaded into the testing rod and then lowered onto the base-plate. The gap thickness (the distance between the top and bottom rheometer plates) was decreased until the biofilm was compressed with an initial normal force of $0.1 \mathrm{~N}$ (an equivalent of a normal stress of $4.9 \times 10^{-4} \mathrm{~Pa}$ over a $25 \mathrm{~mm}$ diameter disk). In previous experiments we determined that $0.1 \mathrm{~N}$ was the lowest compressive force that could be applied to eliminate slippage due to insufficient contact between biofilm and the lower rheometer base-plate (Towler et al., 2003). Slippage was readily evident as sporadic jumps in strain during testing. The application of a consistent initial normal force allowed the gap thickness to vary with biofilm thickness and established a standardized testing method. Biofilm samples, sandwiched between two disks in the rheometer, were allowed to undergo an "adjustment" period of $5 \mathrm{~min}$ after the normal force was applied, to minimize the effects of the normal load application and allow the biofilm to "stabilize" within the rheometer. Over the period of the creep testing $(20 \mathrm{~min})$ the measured normal force dissipated by $32 \pm 15 \%(n=7)$.

To determine the quasi-static creep response of biofilms subjected to constant shear stresses, $S$. mutans biofilms were tested over a range of stresses from 0.05 to $55 \mathrm{~Pa}$.
A total of 49 creep tests were performed using 12 biofilm samples from 6 independent experiments. Further, in a separate set of dynamic experiments, the response of biofilms to harmonic oscillations was studied. The experiments were performed in strain-controlled mode at low strains, i.e. $0.1 \%, 0.125 \%$ and $0.25 \%$, to ensure that the biofilm response remained in the linear region characterized by direct stress-strain proportionality. The effects of cyclic frequencies on the time-dependent response of biofilms were studied by sweeping across a frequency range $10^{-1}$ to $10^{2} \mathrm{~Hz}$. The parameters measured in the dynamic experiments included the complex shear modulus and viscosity as a function of frequency.

\section{Microscopy}

To characterize and quantify the structure of the biofilms grown on the rheometer disks, microscopic examinations were performed using confocal scanning laser microscopy (CSLM) (Leica TCS-NT). Biofilms were stained directly on the rheometer disks with the BacLight live/dead kit (Molecular Probes, Eugene, OR) using $1.5 \mu \mathrm{l} \mathrm{SYTO9+}$ $1.5 \mu \mathrm{l}$ propidium iodide per milliliter of nanopure water and incubated for $1 \mathrm{~h}$ before visualization (488 nm excitation wavelength). Confocal stacks were collected to visualize the three-dimensional structure of the biofilm. The average biofilm thickness was found from taking measurements at 10 random locations on each of three rheometer disks. To characterize the effect of the compressive force applied prior to rheological testing on biofilm structure preweighed glass slides and coverslips were placed on top of a biofilm colonized disk to achieve a force of $0.1 \mathrm{~N}$. The biofilm was then again visualized with CSLM and the thickness remeasured. 


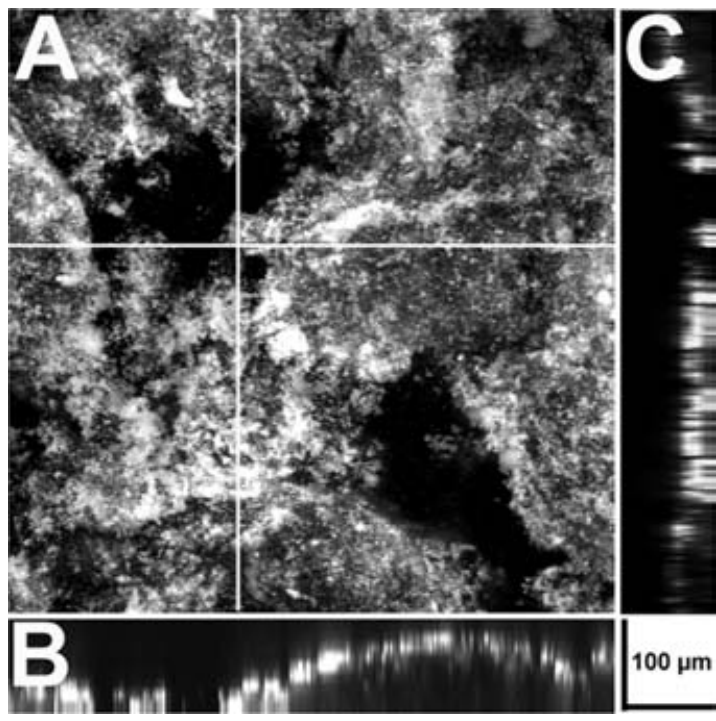

Fig. 3: CSLM image of $S$. mutans biofilm grown on a HA-coated rheometer disk: (A) plan view; (B) and (C) cross-sections. Scale bar represents $100 \mu \mathrm{m}$.

\section{RESULTS AND DISCUSSION}

\section{Biofilm structure}

Biofilms grew readily on the HA-coated rheometer disks and formed a heterogeneous structure consisting of tower- and "mushroom"-shaped cell clusters separated by interstitial water channels (Fig. 3). The mean thickness of the biofilm was $162 \mu \mathrm{m}$, with a standard deviation (SD) of $73 \mu \mathrm{m}, 45 \%$ of the mean $(n=10)$. When the biofilms were compressed with $1 \mathrm{~N}$ normal force (Fig. 4) the mean thickness was reduced to $102 \mu \mathrm{m}$ and the SD to $10 \mu \mathrm{m}$, or $10 \%$ of the mean, reflecting a more uniform thickness (Fig. 5). When plate-type geometries are used for testing, some degree of compression is inevitable. Although we used the minimum amount of compression required to avoid slip, compression may still influence the mechanical characterization of the biofilm. Since the biofilms were unconfined during compression and water is non-compressible it is expected that water would be "squeezed" out of the macropores and channels in the biofilm, as appeared to be the case from the CSLM images. For example, in Fig. 4 the biofilm porosity was decreased from approximately 0.33 in the uncompressed state to 0.14 after compression. The dewatering from biofilm macropores during compression will result in the measured mechanical properties being more representative of the biomass proportion (cells and extracellular polymeric substance (EPS) matrix) of the biofilm. The compression of biofilm thickness by $37 \%$ in the present study compares with the $21 \%$ compression caused by increased fluid shear in a flow cell (Stoodley et al., 1999b). To obtain meaningful interpretation, especially of a challenging material such as biofilm, which is thin, structurally variable and heterogeneous in composition, it is important to develop standardized
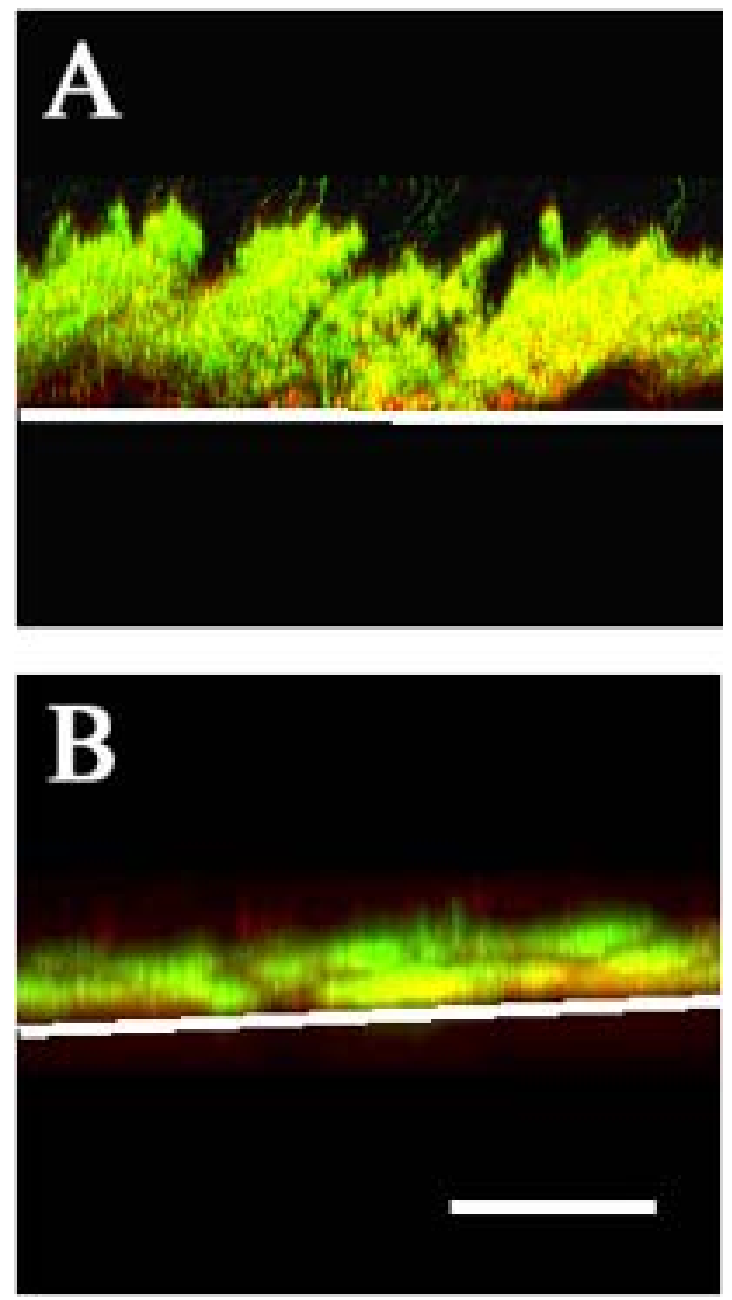

Fig. 4: Cross-section of biofilm on a HA-coated rheometer disk: (A) uncompressed biofilm; (B) biofilm compressed with $0.1 \mathrm{~N}$ normal force. Scale bar respresents $200 \mu \mathrm{m}$.

testing conditions and also to determine the effect that the test itself may have on the physical structure.

\section{Viscoelastic properties of S. mutans biofilms}

\section{Creep response}

The results of quasi-static creep tests demonstrated that the response of biofilms under sustained shear stresses was time dependent. The creep and recovery curves demonstrated the typical behavior of a viscoelastic material, which combines the properties of elastic solids and viscous fluids (Fig. 6). This type of behavior is observed in a variety of materials, including biological materials (Fung, 1993) and organic polymers (Wineman \& Rajagopal, 2000).

The creep behavior of the $S$. mutans biofilms was characterized by a viscoelastic constitutive law in the form

$$
\gamma(t)=\tau(0) J(t)+\int_{0}^{t} J(t-s) \dot{\tau}(s) \mathrm{d} s
$$




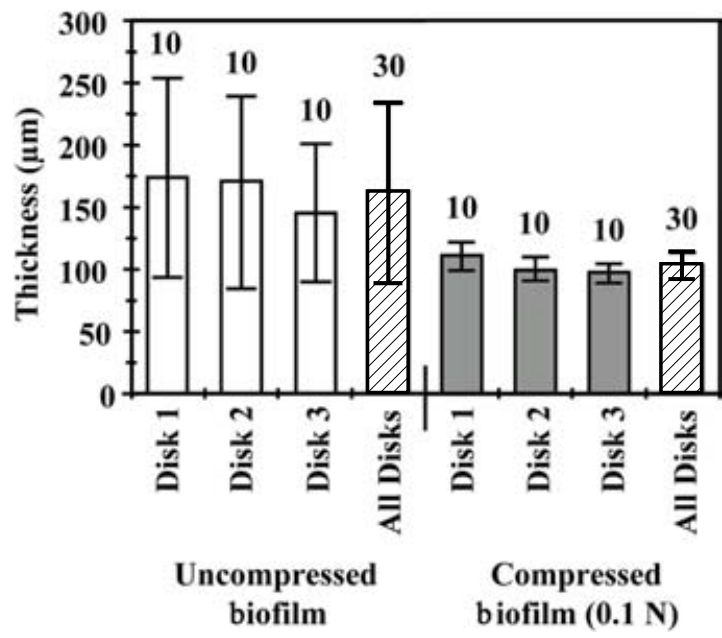

Fig. 5: Thickness in biofilm before and after compression with $0.1 \mathrm{~N}$ normal force. Bars indicate $1 \mathrm{SD} ; n$ is given over the bar. Mean and SD of thickness measured at 10 locations on each of three disks (solid bars) in the rheometer are shown to illustrate variability on and between disks. Mean thickness for all disks is indicated by hatched bars. There was no significant difference between the thickness of biofilms grown on the three disks $(P>0.41)$.

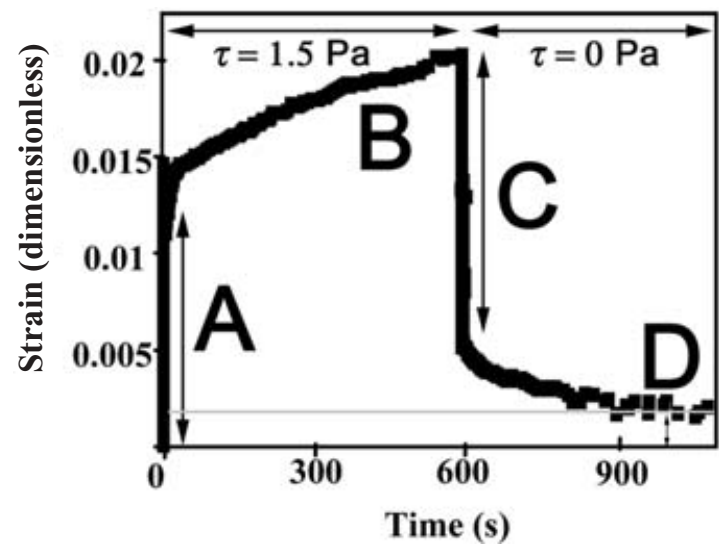

Fig. 6: Creep and recovery of a $S$. mutans biofilm. A shear stress of $1.5 \mathrm{~Pa}$ was applied for $300 \mathrm{~s}$ and then removed. The curve shows four characteristic regions of a viscoelastic material. (A) Instant elastic strain. The effective shear modulus $G$ can be found by dividing the applied shear stress by the strain. (B) Viscous flow region. The effective viscosity $\eta$ can be found by dividing the applied shear stress by the linear region of the strain rate which is the slope of the curve. (C) Instantaneous elastic recovery on removal of the shear stress. (D) Residual strain due to viscous flow.

where $\gamma$ and $\tau$ denote shear strain and shear stress respectively, $\dot{\tau}$ is the stress rate; and $J(t)$ represents the creep compliance, which characterizes the material response as a function of time $t$. The creep compliance $J(t)$ can be obtained experimentally by measuring the material response under a constant stress $\left(\tau_{\mathrm{o}}\right)$ so that $J(t)$ represents a normalized creep curve according to

$$
J(t)=\gamma(t) / \tau_{\mathrm{o}}
$$

The normalized creep response of a biofilm tested for $300 \mathrm{~s}$ under sustained shear stresses $\tau_{\mathrm{o}}$, ranging from 0.75

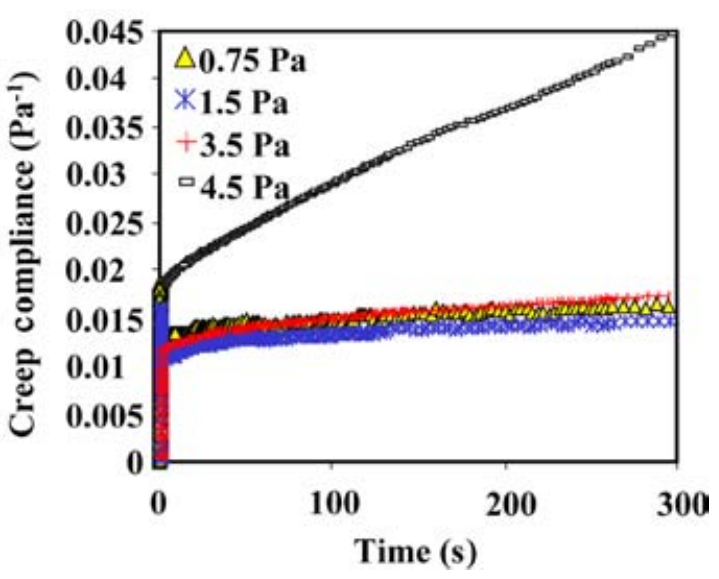

Fig. 7: Linear and non-linear viscoelastic response of $S$. mutans biofilm demonstrated by running creep tests at increasing shear stress. The creep compliance $(J)$ is the strain divided by the applied shear stress. At shear stresses up to $3.5 \mathrm{~Pa}$ the curves overlie, indicating a linear response to shear. The effective viscosity in this region was approximately $1 \times$ $10^{5} \mathrm{~Pa}$. The departure of the curve at a shear stress of $4.5 \mathrm{~Pa}$ indicated that the biofilm was in the non-linear region. The effective viscosity was reduced to $7 \times 10^{3} \mathrm{~Pa} \mathrm{~s}$, indicating that the biofilm may have exceeded a yield shear. Data from creep tests run at 0.5 and $2.5 \mathrm{~Pa}$ were omitted from the graph because they also overlaid the $0.75,1.5$ and $3.5 \mathrm{~Pa}$ curves, causing difficulty in distinguishing individual curves.

to $4.5 \mathrm{~Pa}$, is shown in Fig. 7. The biofilm exhibited a stressindependent viscoelastic behavior below shear stresses of 3.5 Pa. At higher stresses the creep rates tend to increase substantially, implying that, at stresses below $\tau_{\mathrm{o}}=3.5 \mathrm{~Pa}$, the biofilm could be treated as a linearly viscoelastic material. At stresses higher than this linearity limit the material response becomes stress dependent and must be treated as non-linearly viscoelastic.

It follows from equation 2 that, at time $t=0$, the instantaneous "effective" shear modulus of the material can be determined from $G_{0}=G(0)=\gamma(0) / \tau_{\mathrm{o}}$. The effective viscosity $(\eta)$ was determined from the linear region of the slope, which for these experiments was taken as being between $t=180 \mathrm{~s}$ and $t=300 \mathrm{~s}$. The mean $G_{0}$ and $\eta$ from 37 biofilm samples taken from 6 independent experiments were $1.9 \pm 3.8 \times 10^{2} \mathrm{~Pa}$ and $2.8 \pm 6.4 \times 10^{5} \mathrm{Pas}$, respectively. It is important to note that, in general, the instantaneous properties of viscoelastic materials depend on the rate of loading. Therefore, the effective shear modulus determined from creep experiments cannot be treated as a precise material characteristic. Nevertheless, such experiments provide a reasonable estimate regarding the response of viscoelastic materials at the time of the load application.

\section{Dynamic response}

When a linear viscoelastic material is subjected to a history of sinusoidal strain

$$
\gamma(t)=\gamma_{\mathrm{o}} \sin \omega t
$$

the respective stress is obtained in the form (Wineman \& Rajagopal, 2000)

$$
\tau(t)=\gamma_{\mathrm{o}}\left[G^{\prime}(\omega) \sin \omega t+G^{\prime \prime}(\omega) \cos \omega t\right]
$$




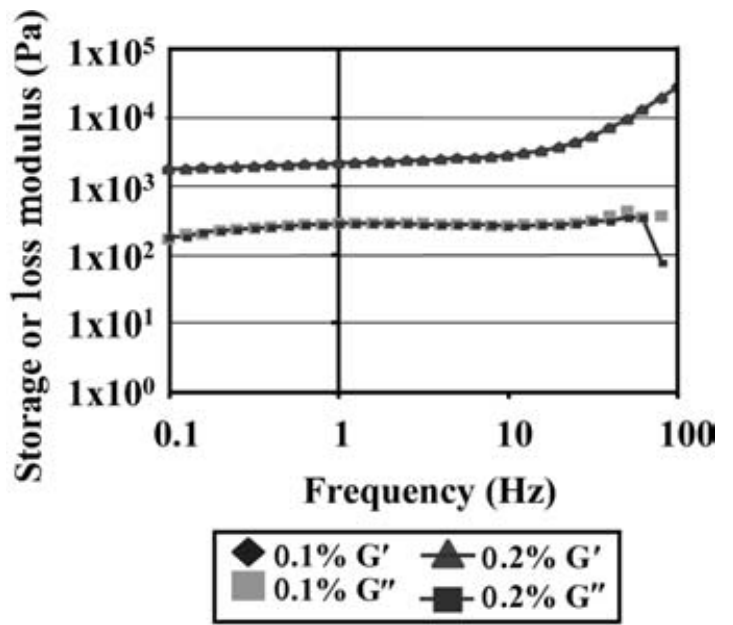

Fig. 8: Dynamic testing of $S$. mutans biofilm showing storage and loss moduli as a function of frequency at two different strains $\left(\gamma_{0}\right)$ of $0.1 \%$ and $0.2 \%$

where $\gamma_{0}$ is the strain amplitude, and $G^{\prime}(\omega)$ and $G^{\prime \prime}(\omega)$ represent, respectively, the storage and loss moduli as functions of frequency $\omega$. The storage modulus, $G^{\prime}(\omega)$, characterizes the ability of the material to store energy, whereas the loss modulus, $\mathrm{G}^{\prime \prime}(\omega)$, characterizes energy dissipation in the material under dynamic excitations.

An additional useful material characteristic derived from dynamic experiments is the complex viscosity. The absolute value of the complex viscosity $\left|\eta^{*}\right|$ is defined as (Menard, 1999)

$$
\left|\eta^{*}\right|=\frac{\sqrt{\left(G^{\prime}\right)^{2}+\left(G^{\prime \prime}\right)^{2}}}{\omega}
$$

This parameter characterizes the tendency of the material to flow. The dynamic response of the biofilms in terms of the storage $G^{\prime}(\omega)$ and loss moduli $G^{\prime \prime}(\omega)$ from dynamic frequency sweep experiments, involving strain oscillations at amplitudes $0.1 \%$ and $0.2 \%$ showed good consistency and an insensitivity of $G^{\prime}(\omega)$ and $G^{\prime \prime}(\omega)$ to strain amplitude variations within the linear viscoelastic deformation range (Fig. 8). Further, the absolute value of the complex viscosity $\left|\eta^{*}\right|$ of the biofilms was determined, from frequency sweep experiments at the strain amplitudes $\gamma_{\mathrm{o}}=0.1 \%, 0.125 \%$ and $0.25 \%$, and a normal force of $0.1 \mathrm{~N}$, using equation 5 (Fig. 9). The absolute value of $\left|\eta^{*}\right|$ tended to decrease with increasing frequency $\omega$. Similarly to the results presented in Fig. 8, the value of $\left|\eta^{*}\right|$ showed little sensitivity to the variations of the strain amplitude within the linear viscoelastic deformation range.

It is of interest to compare the latter results, obtained for the $S$. mutans biofilms with the observed dynamic behavior of other rheological materials. First, it is important to note that the diagrams shown in Fig. 9 were qualitatively very similar to those determined for polymeric fluids and melts (Menard, 1999). For the latter materials, a direct correlation has been established between their complex viscosity as a function of frequency

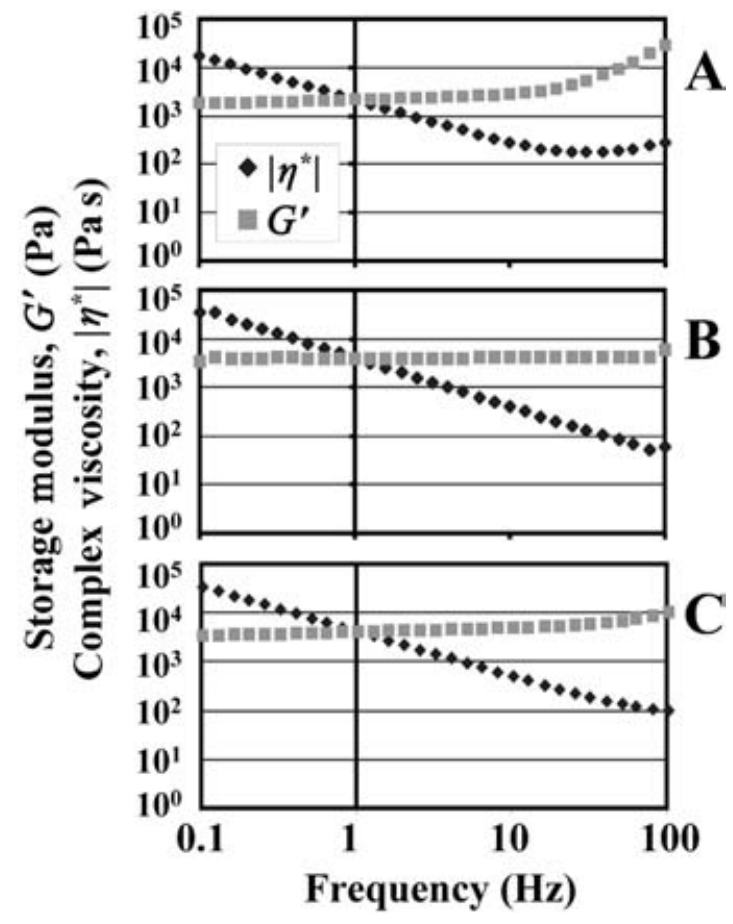

Fig. 9: Dynamic testing of $S$. mutans biofilm at (A) $\gamma_{0}=0.1 \%,(B) \gamma_{0}=$ $0.125 \%$, and (C) $\gamma_{\mathrm{o}}=0.25 \%$.

and the respective changes of their microstructure and molecular weight in response to sinusoidal oscillations. The decrease of the absolute value of complex viscosity $\left|\eta^{*}\right|$ as a function of frequency, observed in the case of the $S$. mutans biofilms, is generally associated with shear thinning.

\section{Constitutive modeling of $\mathbf{S}$. mutans biofilms}

Within the framework of viscoelasticity theory a distinction is made between viscoelastic solids and viscoelastic fluids. A viscoelastic solid subjected to a fixed deformation usually responds with a corresponding component of stress that remains non-zero as long as the deformation is maintained. In contrast, a viscoelastic or rheological fluid under similar conditions produces a stress state that tends to decay to zero. In other words, a viscoelastic fluid exhibits an unlimited number of undeformed configurations, whereas a viscoelastic solid may have only a single equilibrium configuration (Lakes, 1998) and (Christensen, 1971). From the creep curves it was apparent that the behavior of $S$. mutans biofilms could be represented by a viscoelastic constitutive model constructed from springs (elastic elements) and dashpots (viscous elements) (Flugge, 1975). We modeled the viscoelastic behavior of the $S$. mutans biofilms with a four-element Burger model (Fig. 10). This model is commonly used for viscoelastic materials and has recently been used to model pond water biofilms (Towler et al., 2003). The creep compliance modeled by the Burger model correlated well with the experimental data (Fig. 11). 


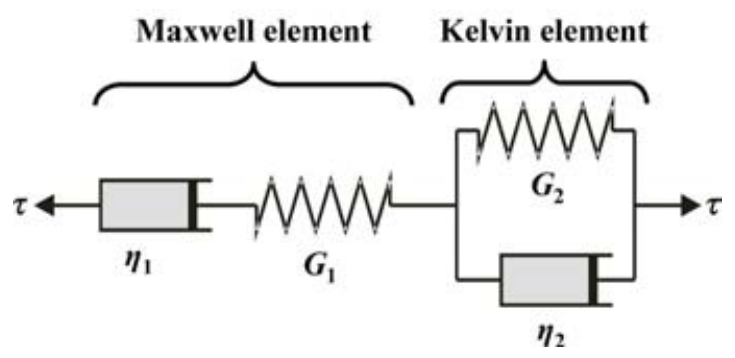

Fig. 10: Burger model representation of a viscoelastic materia constructed from springs with elastic parameters, $G_{1}$ and $G_{2}$, and dashpots (viscous elements that can act as vibration dampners) with viscous parameters $\eta_{1}$ and $\eta_{2}$.

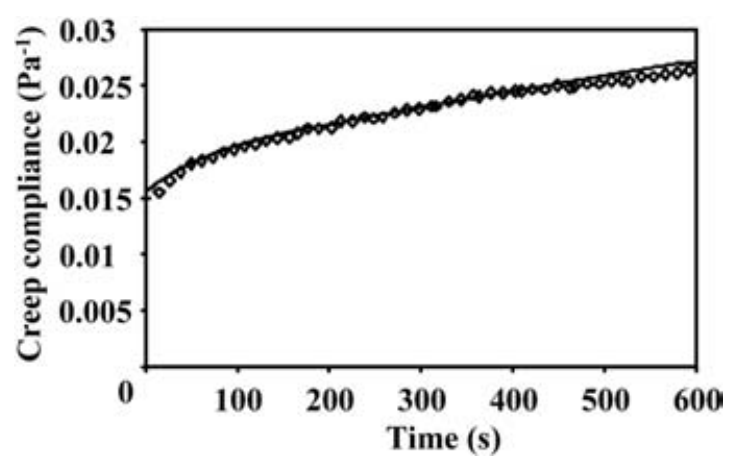

Fig. 11: Creep compliance of $S$. mutans biofilm modeled by a Burger model with parameter values of $G_{1}=63.9 \mathrm{~Pa}, G_{2}=283 \mathrm{~Pa}$, $\eta_{1}=75534 \mathrm{~Pa}$ s and $\eta_{2}=19028 \mathrm{~Pa}$ s. Open circles, experimental data; solid line, model data.

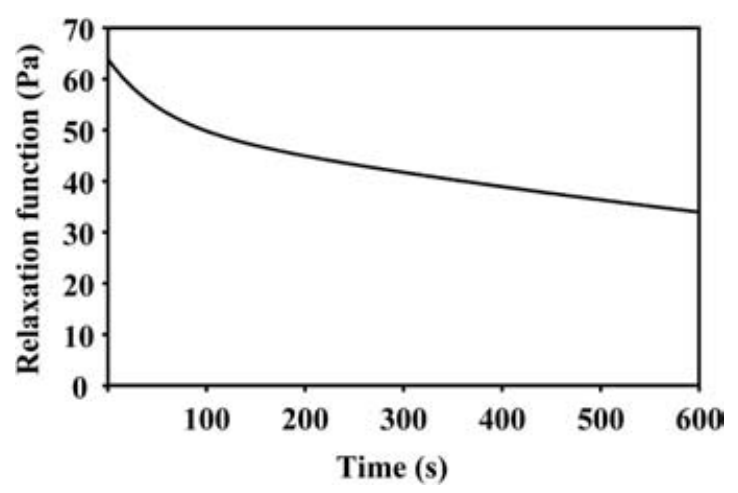

Fig. 12: Computed stress relaxation function of $S$. mutans biofilm derived from the Burger model of the creep compliance curve shown in Fig. 11.

The stress relaxation function of the $S$. mutans biofilm was determined analytically from the model (Fig. 12).

\section{CONCLUSIONS}

The investigation presented in this paper concerns the mechanical properties of bacterial biofilms formed from the dental plaque colonizer $S$. mutans. A consistent experimental programme, based on ASTM standards, was developed to prepare and test the response of biofilms under static and dynamic loading conditions, using a TA Instruments AR 1000 rheometer. The study demonstrated that $S$. mutans biofilms exhibited creep behavior in response to constant shear stresses. The instantaneous effective shear modulus of $S$. mutans ranged from 80 to $200 \mathrm{~Pa}$. It is important to underscore that the magnitude of this mechanical characteristic depends on the loading rates, and therefore cannot be determined with high precision using the described experimental procedure.

Within certain limits, S. mutans biofilms can be treated as linearly viscoelastic materials. Experiments indicate that, at stresses above the viscoelastic linearity limit at $3.5 \mathrm{~Pa}$, creep rates of the biofilms increase considerably, producing stress-dependent non-linear effects, ultimately leading to mechanical destruction of biofilms owing to creep failure. Dynamic experiments involving frequency sweeps in the range $10^{-1}$ to $10^{2} \mathrm{~Hz}$ allowed initial characterization of the behavior of $S$. mutans in response to sinusoidal oscillations. The dynamic response of the biofilms was characterized in terms of the shear storage and loss moduli and complex viscosity. The shear storage and loss moduli of $S$. mutans showed little sensitivity to frequency and amplitude effects within the experimental range of parameters, whereas the dynamic viscosity decreased in response to increasing oscillation frequencies. The latter behavior is similar to that of many polymeric fluids and is often associated with shear thinning of the material. Using the constitutive law, provided by the Burger model, the stress relaxation function of a $S$. mutans biofilm was determined analytically. The $S$. mutans biofilms appeared to exhibit remarkable resemblance to the response of synthetic polymers (Janmey et al., 1990) and other biological fluids such as saliva, mucus, sputum, synovial fluids and protoplasm (Fung, 1993). It is evident that qualitative characterization of the mechanical properties of biofilms is critical in terms of enhancing the understanding of their biological functions, survival mechanisms and surface attachment processes. On this basis, innovative strategies for more effective mechanical removal of pathogen containing biofilms can be developed in dental and medical fields.

\section{ACKNOWLEDGEMENTS}

This work was funded by Philips Oral Healthcare, Inc., Snoqualmie, Washington, USA, and in part by the National Institute of Health RO1 grant GM60052. For their technical advice and contributions we thank: Douglas Dudgeon, Chris McInnes and Gregory Peterson from Philips Oral Healthcare; Isaac Klapper and Todd Shaw from the Center for Biofilm Engineering and Department of Mathematics, Montana State UniversityBozeman; and for the Burger model code Brett Towler 
from the Center for Biofilm Engineering and Department of Civil Engineering, Montana State UniversityBozeman.

\section{REFERENCES}

Adams, H., Winston, M. T., Heersink, J., Buckingham-Meyer, K. A., Costerton, W. J. \& Stoodley, P. (2002) Development of a laboratory model to assess the removal of biofilm from interproximal spaces by powered tooth brushing. American Journal of Dentistry 15, 12B-17B

Christensen, R. M. (1971) Theory of Viscoelasticity: An Introduction, 2nd edn. New York: Academic Press

Christersson, C. E., Glantz, P. O. \& Baier, R. E. (1988) Role of temperature and shear forces on microbial detachment. Scandinavian Journal of Dental Research 96, 91-98

Christersson, C. E., Dunford, R. G., Glantz, P. O. \& Baier, R. E. (1989) Effect of critical surface tension on retention of oral microorganisms. Scandinavian Journal of Dental Research 97 , 247-256

Flugge, W. (1975) Viscoelasticity. New York: Springer-Verlag

Fung, Y. C. (1993) Biomechanics: Mechanical Properties of Living Tissues, 2nd edn. New York: Springer-Verlag

Gift, H. C. (1986) Current utilization patterns of oral hygiene practices. In Dental Plaque Control Measures and Oral Hygiene Practices, pp. 39-71. Edited by H. Löe \& D. V. Kleinman. Oxford: IRL Press

Heersink, J., Costerton, W. J. \& Stoodley, P. (2003) Influence of the Sonicare ${ }^{\circledR}$ toothbrush on the structure and thickness of laboratory grown Streptococcus mutans biofilms assessed by digital time-lapse and confocal microscopy. American Journal of Dentistry 16, 79-83

Janmey, P. A., Hvidt, S., Lamb, J. \& Stossel, T. P. (1990) Resemblance of actin-binding protein/actin gels to covalently crosslinked networks. Nature 345, 89-92
Klapper, I., Rupp, C. J., Cargo, R., Purevdorj, B. \& Stoodley, P. (2002) A viscoelastic fluid description of bacterial biofilm material properties. Biotechnology and Bioengineering $\mathbf{8 0}$, 289-296

Koerstgens, V., Flemming, H.-C., Wingender, J. \& Borchard, W. (2001) Influence of calcium ions on the mechanical properties of a model biofilm of mucoid Pseudomonas aeruginosa. Water Science and Technology 43, 49-57

Kuramitsu, H. K. (1993) Virulence factors of mutans streptococci: role of molecular genetics. Critical Reviews in Oral Biology and Medicine 4, 159-176

Lakes, R. S. (1998) Viscoelastic Solids. Boca Raton, FL: CRC Press

Marsh, P. D. (2000) Oral ecology and its impact on oral microbial diversity. In Oral Bacterial Ecology, pp. 11-65. Edited by H. K. Kuramitsu \& R. P. Ellen. Wymondham, UK: Horizon Scientific Press

Menard, K. P. (1999) Dynamic Mechanical Analysis. Boca Raton, FL: CRC Press

Moore, L. V. H., Moore, W. E., Cato, E. P., Smibert, R. M., Burmeister, J. A., Best, A. M. \& Ranney, R. R. (1987) Bacteriology of human gingivitis. Journal of Dental Research 66 , 989-995

Stoodley, P., Lewandowski, Z., Boyle, J. D. \& Lappin-Scott, H. M. (1999a) The formation of migratory ripples in a mixed species bacterial biofilm growing in turbulent flow. Environmental Microbiology 1, 447-457

Stoodley, P., Lewandowski, Z., Boyle, J. D. \& Lappin-Scott, H. M. (1999b) Structural deformation of bacterial biofilms caused by short term fluctuations in flow velocity: an in-situ demonstration of biofilm viscoelasticity. Biotechnology and Bioengineering 65, 83-92

Towler, B. W., Rupp, C. J., Cunningham, A. B. \& Stoodley, P. (2003) Viscoelastic properties of a mixed culture biofilm from rheometer creep analysis. Biofouling 19, 279-285

VanHoute, J. (1980) Bacterial specificity in the etiology of dental caries. International Dental Journal 30, 305-326

Wineman, A. S. \& Rajagopal, K. R. (2000) Mechanical Response of Polymers. New York: Cambridge University Press. 